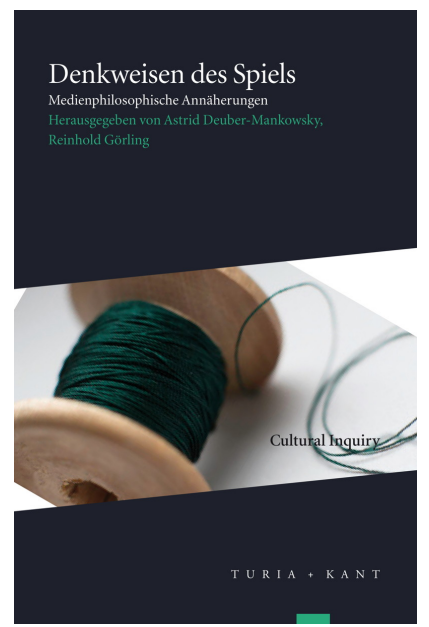

Denkweisen des Spiels: Medienphilosophische Annäherungen, hg. v. Astrid Deuber-Mankowsky und Reinhold Görling, Cultural Inquiry, 10 (Wien: Turia + Kant, 2017), S. 191-212

\section{LISA HANDEL}

\section{Irdisches Spiel - »Queer messmates in mortal play«}

\author{
ZITIERVORGABE:
}

| Lisa Handel, 》Irdisches Spiel - »Queer messmates in mortal play«《, in Denkweisen des Spiels: Medienphilosophische Annäherungen, hg. v. Astrid Deuber-Mankowsky und Reinhold Görling, Cultural Inquiry, 10 (Wien: Turia + Kant, 2017), S. 191-212 <https://doi.org/10. 37050/ci-10_11>

\section{ANGABE ZU DEN RECHTEN:}

(C) by the author(s)

This version is licensed under a Creative Commons AttributionShareAlike 4.0 International License. 


\section{IRDISCHES SPIEL - »QUEER MESSMATES}

IN MORTAL PLAY «1

Lisa Handel

\section{ZOO CITYS DIFFRAKTIONELLER CYBERPUNK: FADENSPIELE SUBALTERNER SYMBIONTEN ${ }^{2}$}

Die düstere Szenerie von Lauren Beukes Speculative Fiction-Urban-NoirRoman Zoo City führt in ein fiktives zeitgenössisches Johannesburg, das von dem sogenannten Getierten-Phänomen gezeichnet ist. Das Getierten-Phänomen, pejorativ die Zoo-Pest, im wissenschaftlichen Fachjargon Erworbene Symbiontenfreie Gewöhnung (Aquired Aposymbiotic Familiarism, kurz AAF), wird in Zoo City vorgestellt als ein Phänomen des Auftretens und der Ausbreitung prekärer symbiontischer Existenzweisen zwischen menschlichen und nichtmenschlichen Tieren, die in ihrer Verbindung magische Fähigkeiten hervortreten lassen, gleichzeitig jedoch ständig bedroht sind von ihrer existenziellen Auslöschung durch den sogenannten Sog (die schwarze Wolke), der als unausweichliches Schicksal ihres Endes die Aposymbionten erwartet. Aber das Prekärsein

1 Donna Haraway, When Species Meet (Minneapolis: University of Minnesota Press, 2008), S. 19.

2 Unter Diffraktion verstehe ich mit Donna Haraway und Karen Barad eine kritische Methode, die jedoch die kritische Schreibpraxis impliziert, dass es keine unschuldigen Schreibpraktiken, keinen unberührten Ort außerhalb, keinen pseudo-göttlichen Standpunkt gibt, von dem aus man das Geschehen unberührt überblicken würde, stattdessen geht es darum, den niemals unschuldigen Verstrickungen immer weiter zu folgen, um-zu-stricken um derart effektive Verschiebungen zu erzeugen. Der Begriff der Diffraktion (Beugung) entstammt der physikalischen und nicht der geometrischen Optik, er bezieht sich also nicht auf Reflexion, sondern bezeichnet eine mediale Anordnung, in der Wellen auf ein Hindernis treffen und ein Interferenzmuster erzeugen. »Die Beugung bringt nicht - wenngleich verschoben - das Selbe hervor, wie Spiegelung und Brechung es tun. Die Beugung bildet die Überlagerung ab, nicht die Replikation, Spiegelung oder Reproduktion. Ein Beugungsmuster verzeichnet nicht den Ort, wo Differenzen auftreten, sondern den Ort, wo die Wirkungen der Differenz erscheinen. « (Donna Haraway, Monströse Versprechen. Die Gender- und Technologie-Essays (Hamburg: Augment Verlag, 2006), S. 21). 
der Getierten gründet hier nicht nur in ihrer Ausgesetztheit gegenüber dem Sog, schwerer wiegt wohl ihre soziale, politische und juridische Prekarisierung. In der Welt von Zoo City regieren jene auf Ausschluss zielenden Diskurse der Verwerfung, die besagen, dass nur "Schuldige« das Getierten-Phänomen auf sich ziehen.

Wer Schuld auf sich lädt [...] muss mit zwei Dingen leben: einer magischen Begabung. Und einem Tier, das plötzlich da ist und einem nie mehr von der Seite weicht. ${ }^{3}$

Tatsache ist, wir sind alle Verbrecher. Mörder, Vergewaltiger, Junkies. Abschaum. In China erhält jeder Zoo die Todesstrafe, aus Prinzip. Weil nichts auf der Welt so laut `schuldig! r ruft wie ein Geister-Vieh an deiner Seite. ${ }^{4}$

Zurückdatiert auf die 1980er Jahre, in denen die ersten Fälle einer »Epidemie, die nichts mit Krankheit zu tun hat « bekannt wurden, ${ }^{5}$ weist das Getierten-Phänomen aus Zoo City so auch nicht zufällig eine unübersehbare Parallele zur Stigmatisierung der HIV-Erkrankten auf. Zoo City gibt keine Erklärung für das Phänomen; in den intramedialen Gefügen des Buches, die aus Ausschnitten aus (fiktiven) Dokumentarfilm-OnlineArchiven, Blogs, Mails und Twitter-Statements sozialer Netzwerke der hyperkybernetisierten, informations-durchtränkten Welt von Zoo City zusammengesetzt sind, werden verschiedenste Spekulationen über die Getierten verhandelt - angefangen bei der Theorie eines Nuklearunfalls, dem Glauben an die physische Manifestation der Sünden, der Heimsuchung der Cyberculture, Genmanipulation, bis hin zur These der toxischen Wiedergeburt hinduistischer Gläubiger in den seltsamen Seinsmodalitäten postmoderner Welten. So sehr Zoo City einen Grund oder Ursprung für das Symptom dieser sogenannten »ontologischen Verschiebung " verwehrt, ${ }^{6}$ desto mehr stehen hier die wirksamen Bedeutungen von Gewicht für die verkörperten und situierten Lebensformen auf dem Spiel. Staatliche Gewalt und Folter sind dabei nur eine Form der Gewalt unter anderen:

3 Lauren Beukes, Zoo City (Reinbek bei Hamburg: Rowohlt Taschenbuch Verlag, 2015), Klappentext.

4 Ebd., S. 16.

5 Ebd., S. 77.

6 Ebd., S. 81. 
,Unsere Tiere werden in Käfigen in einem anderen Teil des Gefängnisses gehalten. Wir kriegen sie nicht zu Gesicht. Wenn die Wärter uns foltern wollen, dann stecken sie die Tiere hinten in ein Auto und fahren bis nach Keti Bandar. Die Schmerzen sind nicht auszuhalten, du schreist, du kotzt, und du gibst alles zu, was sie wollen. Meine Kobra war bei mir, als ich festgenommen wurde. Da war ich neun. Die Polizei hat mich von der Straße weggegriffen, als ich mit der Kobra rumgelaufen bin. Die haben behauptet, ich wäre in ein Haus eingebrochen. Das stimmt nicht, aber die haben mich verprügelt, bis ich gesagt habe, ich war's. [...] Ich habe meine Kobra nicht gesehen, seit ich festgenommen wurde. Ich bin jetzt 14 Jahre alt.< Auszug aus Hinter Gittern: Getiert und weggesperrt, Fotos und Interviews: Steve Deacon, HarperCollins $2008^{7}$

Im Kontrast zu den hochfinanzierten technowissenschaftlichen Programmen einiger geopolitischer Größen - »In den USA, Australien, Iran, an solchen Orten, checken sie einen von Kopf bis Fuß durch, Kernspintomographie von Körper und Gehirn, Hormonanalyse, das volle Programm ${ }^{8}$ - lässt das Johannesburg aus Zoo City, die Aposymbionten in Hinsicht auf die medizinische Behandlung/Pathologisierung weitgehend, wohl aus finanziellen Gründen, in Ruhe, gettoisiert diese jedoch in der Zoo-City und errichtet zugleich Apo-freie-geschützte Gated-Communitys für die ungetierte, zumeist weiße, Bevölkerung. Dabei reicht die Bandbreite der auf Prozessen des »Othering « gründenden, distinktiven Bezugsweisen der ungetierten Bevölkerung auf die Andersheit der Getierten, wie man u.a. Blog-Einträgen entnehmen kann, von exotistischer Fetischisierung der Art »Verbotene Liebe! Meine Zoo-Romanze «" oder » Geile Seite für kostenlose Zoo-P0rno gefunden!!! «, ${ }^{10}$ der medialen Sensationalisierung von vermeintlichem Tiersex und Tieropferungen, der Vermarktung von getierten Hyänen-Rappern und egoshootermäßigen Videospielen a la "Zootopia ", ${ }^{11}$ in denen »harte Kerle mit knurrenden Panthern « alles niedermetzeln, was ihnen in den Weg gerät, bis hin zu extremen Formen von Rassismen und Faschismen und Hate Speech im Netz:

\footnotetext{
Ebd., S. 101-02.

Ebd., S. 153.

Ebd., S. 71.

Ebd., S. 79.

Ebd., S. 108.
} 
Leute, kapiert ihr's nicht, Apos sind nicht menschlich. Wie der Name schon sagt: Zoos. Getierte. Aposymbionten. Symbiontenfreie. Oder welches Wort sonst gerade pc ist. Apo wie snicht menschlich`. Wie die Abkürzung von >Apokalypse ‘. Das gehört alles zum Tarnkappenbomberkrieg, der sich als Kampf für Apo-Rechte tarnt, sich aber in Wahrheit gegen anständige Bürger richtet. Steht im fünften Buch Moses: Darum sollst du solchen Gräuel nicht in dein Haus bringen, damit du nicht dem Bann verfällst wie jene, sondern du sollst Ekel und Abscheu davor haben; denn es steht unter dem Bann. Und Exodus: Eine Hexe sollst du nicht am Leben lassen. Muss ich es noch einzeln buchstabieren? Höllensog. Bann des Gräuels. Gott ist gnädig, aber nur zu richtigen, echten, REALEN menschlichen Wesen. Apos sind Verbrecher. Abschaum. Das sind nicht mal Tiere. Die sind nicht mal Lebewesen und die werden das kriegen, was $[\mathrm{MEHR}]^{12}$

Die Andersheit des Getiertseins intersektioniert hier mit vielfältigen anderen Markierungen von subalterner Alterität - wobei »Hautfarbe « als eine der bedeutendsten Marker erscheint. So ist es kein Zufall, dass die Hauptprotagonistin Zinzi December farbig, weiblich und getiert ist, wobei keine dieser Differenzen ineinander aufgeht. Die vormals drogensüchtige Zinzi glaubt ihren Bruder ermordet zu haben und seitdem trägt sie das pelzige, schwere, oftmals nörgelnde Wesen mit den langen Armen um ihre Schultern geschlungen ununterbrochen mit sich - Faultier. Faultier und Zinzi erfüllen nicht die Heldenrolle der rebellischen Widerstandskämpfer gegen »das Regime", wie im klassischen Cyberpunk, stattdessen stecken sie bis über alle vier Ohren morasttief im Sumpf der Fadenspiele von Zoo City. In der Symbiose erscheint ein magisches Vermögen - Mashavi -, das die, in der Verbindung erscheinenden, Fähigkeiten eines Aposymbionten mit dem aposymbiontischen Tier bezeichnet. Zinis und Faultiers Mashavi ist das relativ unspektakulär daherkommende Vermögen des Aufspürens verlorener Gegenstände. Das sich hierin eröffnende Gefüge erscheint nicht nur als Symbiose zwischen den beiden Lebewesen, sondern zugleich als Verbindung mit der alltäglichen Dingwelt von Zoo City. Zinzi und Faultier spüren die Verbindungen zwischen verlorenen Objekten und ihren zugehörigen menschlichen Akteuren wie einen Zug: "Rausgehen ist für mich wie mitten in ein Spinnennetz hineinlaufen, oder so, als hätte jemand Garnknäuel im Irrenhaus verteilt und die Insassen aufgefordert, alles mit allem zu

12 Ebd., S. 80. 
verknüpfen. « ${ }^{13}$ Da über diese kaum bezahlte Arbeit des Wiederauftreibens verlorener Gegenstände jedoch kaum »Rands « einzutreiben sind, betätigt sich Zinzi, ganz zu Faultiers Missfallen, zudem als illegale Scammerin im Netz. Ihre Subversivität gründet gerade nicht in einem, auf der Kampfmetaphorik basierenden Modell von Widerstand, sondern in den »kleinen ", minoritären Praktiken und Techniken der Relationierung. So ist die Figuration von Symbiose in Zoo City alles andere als homogen. Symbiotische Relationalität wird hier auf mehrfache Weise eingemeindet und angeeignet unter die Herrschaft des Identischen. Denn während die Tiere für die Majorität der Ungetierten lediglich als verdinglichtes Zeichen der Verwerfung des Anderen fungieren -

IIch habe nur gesagt, dass Sie schon einmal einen Mord begangen haben.

'Beihilfe, hat das Gericht gesagt.<

'Das Ding auf Ihrem Rücken sagt aber etwas anderes.

'Es nennt sich Faultier.

'Es nennt sich Schuld. ${ }^{14}$

- dienen sie umgekehrt für die Getierten in den Zoo-Gettos wiederum nur als (instrumentalisierte) Prothesen männlicher Macht und Souveränität - »Diese Männer im Aufzug trugen ihre Tiere aber nicht wie eine Last [...]. Diese Männer trugen die Tiere wie andere Männer Waffen. « ${ }^{15}$ Zinzi und Faultier erproben hingegen in ihrem symbiotischen Zusammenleben, ihrer subalternen »contact zone «, ${ }^{16}$ nichtmännliche, nichtprothetische Existenzweisen von Relationalität jenseits von Aneignung, "Jenseitige Konversationen ${ }^{17}$ die weder aufgehen in den männlichen Allmachtsfantasmen und Reduktionen der nichtmenschlichen Tiere auf phallische Prothesen der Macht, noch in Verschmelzungsfantasmen. Faultiers und Zinzis Symbiose impliziert ein Antworten auf - und Spiel mit - Alterität und Selbstdifferenz.

Faultier schaut missmutig herum, ausgestreckt auf einem Haufen Cassavablätter am Boden, die ich unten auf dem Markt gekauft habe, um ihn milde zu stimmen (für Mungo habe ich ein Fässchen Kellerasseln

\footnotetext{
Ebd., S. 13.

Ebd., S. 34.

Ebd., S. 65-66.

Haraway, When Species Meet, S. 4.

17 Haraway, Monströse Versprechen, S. 81.
} 
besorgt). Wenn ich Faultier zuhause lassen könnte, würde ich es tun. Aber die Rückkopplungsschlaufe der Trennungsangst tut mörderisch weh. Cracksucht ist gar nichts gegen die Sehnsucht nach deinem Tier. ${ }^{18}$

Zoo City ist eine Geschichte, die von den zeitgenössischen mächtigen Netzen kybernetischer Implosionen und hybrider Welten handelt und ihren inhärenten, unauflöslichen Ambivalenzen. Wie Donna Haraway im Kontext der feministischen Wissenschaftskritik herausgestellt hat, wird mit dem cyberkapitalistischen Macht-Dispositiv der »Netzwerke der Informatik der Herrschaft ${ }^{19}{ }^{9}$ einerseits die Frage nach der Relationalität der Existenz, die sich nicht mehr in die modernistischen Dualismen von Natur und Technik, Organismus und Maschine, Subjekt und Objekt, Innen und Außen, Selbst und Anderem einfügt, radikal exponiert. Andererseits wird Relationalität innerhalb der Metaphorik der universellen Vernetzung des Cyberkapitalismus wiederum eingemeindet in ein durch und durch anthropozentrisches, männliches Allmachtsfantasma im neuen Gewand des sogenannten Technohumanismus, welcher den unverwundbaren Übermenschen durch transzendierende Technologie auftauchen lässt.

Aus einer Perspektive könnte das Cyborguniversum dem Planeten ein endgültiges Koordinatensystem der Kontrolle aufzwingen, die endgültige Abstraktion, verkörpert in der Apokalypse des im Namen der Verteidigung geführten Kriegs der Sterne, die restlose Aneignung der Körper der Frauen in einer männlichen Orgie des Krieges. Aus einer anderen Perspektive könnte die Cyborgwelt gelebte soziale und körperliche Wirklichkeit bedeuten, in der niemand mehr seine Verbundenheit und Nähe zu Tieren und Maschinen zu fürchten braucht und niemand mehr vor dauerhaft partiellen Identitäten und widersprüchlichen Positionen zurückschrecken muss. Der politische Kampf besteht darin, beide Blickwinkel zugleich einzunehmen, denn beide machen sowohl Herrschaftsverhältnisse als auch Möglichkeiten sichtbar, die aus der jeweils anderen Perspektive unvorstellbar sind. Einäugigkeit führt zu schlimmeren Täuschungen als Doppelsichtigkeit oder medusenhäuptige Monstren. Zusammenschlüsse von Cyborgs sind monströs und illegitim. Unter unseren gegenwärtigen politischen Verhältnissen gibt es kaum ein verheißungsvolleres Bild von Widerstand und Vereinigung. ${ }^{20}$

\footnotetext{
18 Beukes, Zoo City, S. 148.

19 Haraway, Monströse Versprechen, S. 48.

20 Donna Haraway, Die Neuerfindung der Natur. Primaten, Cyborgs und Frauen 
Während ein Großteil des klassischen Cyberpunks das technohumanistische Masternarrativ unhinterfragt beibehält, bzw. zugrunde legt, um dieses in einer zweiten, kritischen Bewegung in einer dystopischen Zukunftsvision kulminieren zu lassen, die jedoch gleichzeitig die technohumanistische Reduktion des Anderen auf den Status einer Prothese des Selben, die männliche Kriegs- und Souveränitätsmetaphorik (zumeist innerhalb eines Kampfes der Menschen gegen die Maschinen) fortschreibt und verhärtet, beugt Zoo City als subalterne Geschichte und »the other face of cyberpunk ${ }^{21}$ hingegen das technohumanistische Schema selbst. Indem Vernetzung hier gerade nicht mehr phallisch-prothetisch verhandelt wird, sondern als eine Frage der (niemals unschuldigen) Verstrickung - der Symbiose zwischen Heterogenen, seien sie menschlicher, tierischer oder technologischer Art - erscheint, erzeugt Zoo City eine Art Interferenzmuster: Auf dem Spiel steht jenes »Wir sind immer mitten drin ${ }^{22}$ Haraways, welches die kybernetische Logik der Vernetzung im Rahmen des Technohumanismus und des »Kriegs der Sterne «, ${ }^{23}$ der universalen Kontrolle, Entmaterialisierung und Transzendierung auf die Frage des irdisch-sterblichen Ausgesetztseins, Prekärseins, der Situiertheit, Verantwortlichkeit, Verwundbarkeit und Kontingenz hin streut. ${ }^{24}$

Im folgenden gehe ich der Frage nach, inwiefern das Konzept der Symbiose als eine Art »Trickster ${ }^{25}$ innerhalb des kybernetischen Dispositivs und quer zu ihm zu agieren vermag, inwiefern die Metaphorik der Symbiose als ein Fadenspiel agiert, das in der Lage ist, die Fadenspiele der Netze der Macht umzustricken. Dazu werde ich die von der Biologin Lynn Margulis entwickelte Endosymbiontentheorie und die von ihr in Zusammenarbeit mit James Lovelock konzeptualisierte Gaia-Theorie, die beide auf dem Begriff der Symbiose basieren, einerseits innerhalb des Bezugsrahmens der Kybernetik selbst, der Second Order Kybernetik und den Komplexitätswissenschaften, situieren, um andererseits ihre diffe-

(Frankfurt a.M.: Campus Verlag, 1995), S. 40.

21 Gwyneth Jones, »Zoo City by Lauren Beukes - review« (2011) <http://www.theguardian.com/books/2011/may/14/zoo-city-lauren-beukes-review> [Zugriff: 10.08.2015].

22 Haraway, Die Neuerfindung der Natur, S. 98.

23 Ebd., S. 49.

24 Vgl. Haraway, When Species meet, S. 12 und Haraway, Monströse Versprechen, S.10.

25 Haraway, Monströse Versprechen, S. 18. 
rentielle, gebeugte Wiederkehr im Diskurs eines New Materialism, im Sinne einer transformativen Einschreibung in das kybernetische Regime, herauszustellen. So mobilisieren sowohl Gilles Deleuze und Félix Guattari als auch Haraway Margulis Konzept der Symbiogenese um ein derartiges Streuungsmuster der kybernetischen Netzlogik zu erzeugen, während sich Bruno Latour auf das Gaia-Konzept von Margulis und Lovelock bezieht. Symbiogenese und Gaia fungieren hier im Sinne eines irdischen Spiels als Konzepte eines kreativen, schöpferischen und spielerischen Welt-Werdens. Hieran schließt die Frage nach dem subversiven, diffraktionellen Potential eines derartigen neomaterialistischen, posthumanistischen Spiel- und Relationsbegriffs im Hinblick auf das zeitgenössische kybernetische Machtregime an, das Unvorhersagbarkeit längst in sein Kontrollfantasma eingemeindet und Kreativität zum universalen Paradigma erhoben hat.

\section{SYMBIOGENESE ALS INVOLUTION UND WERDEN-MIT}

Die Biologin Lynn Margulis hat mit der Endosymbiontentheorie eine alternative Konzeption von Evolution entworfen, welche an die Stelle des Überlebens des Stärkeren (survival of the fittest) ein irdisches Spiel (earthly play) setzt. Im Zentrum ihrer Endosymbiontentheorie steht das Konzept der Symbiogenese, das von Haraway für ihre Konzeption von Relationalität als »becoming with ${ }^{26}$ aufgenommen wird, und Eingang findet in Deleuzes und Guattaris Konzept der »Involution « als schöpferischem Werden. ${ }^{27}$ Der Begriff Symbiogenese als evolutionstheoretischer Begriff taucht bereits Anfang des 20. Jahrhunderts auf. Konstantin Mereschkowsky versteht darunter die Entstehung von neuen Geweben, Organen, Organismen und Arten durch langfristige Symbiosen. ${ }^{28}$ Über die Reichweite des Begriffs der Symbiose hinaus, der zunächst einmal eine Praxis und Beziehung zwischen heterogenen Akteuren beschreibt, nämlich das Zusammenleben von Lebewesen unterschiedlicher Art in körperlichem Kontakt, bezieht sich Symbiogenese auf einen ontogenetischen Prozess des Hervorgehens neuartiger Ontologien. Symbiogenese

\footnotetext{
26 Vgl. Haraway, When Species Meet, S. 3.

27 Vgl. Gilles Deleuze und Félix Guattari, Tausend Plateaus. Kapitalismus und Schizophrenie (Berlin: Merve Verlag, 1997), S. 325.

28 Vgl. Lynn Margulis, Die andere Evolution (Heidelberg: Spektrum Akademischer Verlag, 1999), S. 48 u. 59.
} 
bezeichnet die Evolution und Emergenz neuer Lebensformen durch symbiontische Verschmelzung. Margulis' Serielle Endosymbiontentheorie (SET) besagt vereinfacht, dass einzellige prokaryotische Lebewesen miteinander Symbiosen eingingen, woraus die komplexen eukaryotischen Zellen mit Zellkern von Pflanzen und Tieren evolutionär hervorgegangen sind. Dass prokaryotische Bakterien als Plastiden und Mitochondrien dauerhaft in Zellen mit Zellkern eingingen und derart die bakterielle Fremd-DNA im Cytoplasma erklären, ist mittlerweile in der Biologie unumstritten, jedoch sind die weitreichenderen Schlussfolgerungen von Margulis kaum anerkannt: Erstens, dass es sich bei Symbiogenese um ein Prinzip der Artenentstehung handelt. ${ }^{29}$ Zweitens, dass sich hieraus ein radikal andersartiges Bild von Evolution ableitet, nämlich Evolution als ein Werden-Mit, ein Werden zwischen Heterogenen, statt ein auf Exklusion angelegter Kampf zwischen Lebewesen. Während das darwinistische Auswahlprinzip der natürlichen Selektion von außen auf molare Entitäten wirkt, wird bei der Symbiogenese die schöpferische Dimension der Evolution konsequent aus dem Zwischen entwickelt, aus einer (intra-aktiven) Medialität, die nicht im Schema von Entitäten und sekundären Kräften, die auf diese einwirken, aufgeht, sondern als ein bakterielles Spiel (bacterial play) den konstituierten Individuen vorausgeht. Margulis Endosymbiontentheorie basiert dabei auf der Vererbung von cytoplastischen Genen, d.h. Genen, die sich nicht im Zellkern befinden, sondern im Cytoplasma, dem flüssigen Teil der Zelle, in dem Mitochondrien, Chloroplasten und andere Organellen angesiedelt sind. Während die Zellkerngenetik ein neodarwinistisches Verständnis von Evolution als survival of the fittest (Kampf um bessere Anpassung) impliziert, setzt SET dagegen auf Ko-Evolution durch Bündnisse. Evolution ereig-

29 Nach Margulis erfordert der Begriff der Art eine solche Symbiose (als Zusammenkommen Fremder), da es im Reich der Bakterien (Zellen ohne Zellkern), bevor sie sich zu Eukaryonten (Zellen mit Zellkern) zusammenschlossen, überhaupt keine »Arten« gab. Vgl. Lynn Margulis, Die andere Evolution, S. 15. Obwohl Artentstehung aufgrund ihrer Zeitlichkeit kaum empirisch zu beobachten ist, finden sich empirische Beobachtungen zu einer neuen Artbildung bei der Taufliege Drosophila, die gerade deshalb in ihrer Implikation nicht anerkannt wurde, weil hier Symbiose im Spiel war: Es kam zu einer genetischen Trennung, die jedoch »Parasiten « basiert war. "Eldrigde tat diese Beobachtung der Artbildung verächtlich ab, weil dabei Symbiose mit Mikroorganismen im Spiel war! Er hatte wie wir alle gelernt, dass Mikroben Keime sind, und wenn Keime auftreten, hat man keine neue Art, sondern eine Krankheit.« (Ebd., S. 17). 
net sich hier durch Gefüge bakterieller Heterogenität, durch Formen des Zusammenlebens, der Durchdringung und Verknüpfung, die noch nicht der Reproduktionslogik der Zellkern-DNA-Chromosomentheorie folgen - durch Weisen des Mit-Seins. Die Endosymbiontentheorie lässt sich damit als eine Spielart des Neo-Lamarckismus begreifen. ${ }^{30}$ Während der einfache Lamarckismus auf der Vererbung von erworbenen Eigenschaften basiert, legt SET die Erwerbung ganzer Organismen und »fremder « DNA durch Bündnisse dar. Symbiogenese ist Evolution durch Vererbung erworbener Genausstattungen. Damit vereint sie verschiedenartige Individuen zu neuen Lebensformen, die Heterogenes in Gefügen zusammenbringen und derart maschinisch im Sinne Deleuzes und Guattaris operieren. Bereits auf der Ebene einer Zelle tritt damit an die Stelle des »Selben " und Identischen eine unangeeignete Fremdheit und Andersheit, die umso radikaler auf der Ebene des Organismus gedacht werden muss:

To become one is always to become with many [...] I love that when $>\mathrm{I}$ die, all these benign and dangerous symbionts will take over and use what is left of >my< body, if only for a little while, ssince< we are necessary to one another in real time. ${ }^{31}$

Symbiose wird hier nicht als Vereinigung oder Verschmelzung im Sinne der Entstehung einer neuen, mit sich identischen Einheit begriffen, sondern ist auf allen Ebenen der Emergenz, sei es Zelle, Organismus oder Art, in das - niemals in eine Totalität oder Identität zu überführende Spiel des Mannigfaltigen und Differenten deterritorialisiert. So mobilisiert Haraway in When Species Meet Margulis Symbiogenesebegriff als prozesstheoretisches Medienkonzept des »becoming with «, im Sinne einer Relationalität bzw. Intra-Aktivität quer zur konstituierten Welt molarer Entitäten. Auch Deleuze und Guattari beziehen sich auf den Symbiosebegriff als einer transversalen Relation des Mannigfaltigen um ihre maschinische Konzeption des Werdens als »Involution « in Tausend Plateaus zu entfalten. "Jede Mannigfaltigkeit ist symbiontisch und vereinigt in ihrem Werden Tiere, Pflanzen, Mikro-Organismen und verrückte Teilchen, eine ganze Galaxie. « ${ }^{32}$ Den Begriff der Involution entwickeln Deleuze und Guattari derart in ihrer Auseinandersetzung mit den Komplexitätswissenschaften als rhizomatischen Gegenbegriff zur baumarti-

\footnotetext{
30 Vgl. Margulis, Die andere Evolution, S. 17.

31 Haraway, When Species Meet, S. 4.

32 Deleuze und Guattari, Tausend Plateaus, S. 341. 
gen Evolution der Abstammung: Involution ist Evolution durch Bündnisse, statt durch Abstammung.

Wenn der Neo-Evolutionismus seine Originalität unterstreichen konnte, so lag das zum Teil an solchen Phänomenen, bei denen die Evolution nicht vom weniger zum höher Differenzierten geht und nicht länger eine abstammungs- und erbschaftsmäßige Evolution ist, sondern vielmehr kommunikativ oder ansteckend wird. Wir würden diese Form der Evolution, die zwischen Heterogenen abläuft, lieber als >Involution` bezeichnen [...]. Das Werden ist involutiv, die Involution ist schöpferisch. ${ }^{33}$

Derart fungiert Margulis Konzept der Symbiogenese sowohl bei Haraway als auch bei Deleuze und Guattari als eine den Naturbegriff destabilisierende Bewegung - als Spiel »widernatürlicher " Verbindungen..$^{34}$ Symbiogenetische Relationalität fügt sich gerade nicht in den modernistischen Dualismus von Technik und Natur, sondern fungiert wie Haraways »Die Cyborg " als eine Figuration jener »naturalcultural contact zones « ${ }^{35}$ der "Gewebe, Netze, Netzwerke und klebrigen Fasern «, die die Wirklichkeit der zeitgenössischen »implodierten Welten « der kybernetisierten "Neuen Weltordnung $A G$ « zugleich bilden, als auch immanent unterwandern bzw. umgestalten. ${ }^{36}$ Haraway und Deleuze/ Guattari beugen derart auch die komplexitätstheoretische bzw. neokybernetische Modellierung von Emergenz, um diese gerade nicht als Selbstorganisation bzw. "Ordnung aus dem Chaos « zu lesen, sondern um die Realität der transversalen bzw. intra-aktiven Relation selbst zu affirmieren, die in keiner Ordnung aufgeht.

Margulis and Sagan put it more eloquently when they write that to be an organism is to be the fruit of the co-opting of strangers, the involvement and infolding of others [...]. Attraction, merger, fusion, incorporation, co-habitation, recombination - both permanent and cyclical - and all other forms of forbidden couplings, are the main sources of Darwin's missing variation $[\ldots]$. These are also the cobblings together that give meaning to the becoming with of companion species in naturecultures.

\footnotetext{
33 Ebd., S. 325.

34 Ebd., S. 351.

35 Haraway, When Species Meet, S. 7.

36 Haraway, Monströse Versprechen, S. 10.
} 
Cum panis, messmates, to look and to look back, to have truck with: those are the names of my game. ${ }^{37}$

\section{GAIAS FADENSPIELE}

Zusammen mit dem (Atmosphären-)Chemiker, Biophysiker und Mediziner James Lovelock hat Lynn Margulis Mitte der 1960er-Jahre die GaiaHypothese der »lebenden Erde« (Gaia: altgriechisch für »Mutter Erde», Erdgöttin und Große Mutter) formuliert. Sie besagt, dass die Erdoberfläche und Biosphäre ein dynamisches, selbstorganisierendes System bildet, das als lebendig verstanden werden muss. Die Symbiose aller Lebewesen der Erde untereinander und zu ihren anorganischen Umwelten lässt hiernach in einem bottom-up-Prozess eine lebendige Erde emergieren, die durch einen feedback-loop rekursiv in einem top-down-Prozess wiederum die Bedingung bildet für diese Ökologien des Lebens. ${ }^{38}$ Gaia wäre nach Margulis als symbiogenetisches Spiel zwischen Heterogenem zu begreifen, sobald man es vom »bacterial play« auf alle Skalen von Ereignissen bzw. irdischen Ökologien ausdehnt. »Gaia ist einfach Symbiose vom Weltraum aus gesehen. « ${ }^{39}$ Dabei steht Gaia jedoch expliziter in der kybernetischen und systemischen Tradition, insofern hier in problematischer Weise die großen Erzählungen von Einheit, Holismus, Ganzheit und kosmischer Harmonie aufgerufen werden, denen das symbiogenetische, mikroprozessuale Spiel »unheilbare « Differenz entgegensetzt. So basiert Margulis Gaia-Konzeption auf der second order-kyberneti-

Haraway, When Species Meet, S. 31-32.

Dabei entwickelt der Atmosphärenchemiker Lovelock die Gaia-Hypothese der "lebendigen Erde« aus der Frage heraus, wieso die instabilen und unwahrscheinlichen Mischungsverhältnisse von Gasen der Erdatmosphäre überhaupt existieren und sich aufrechterhalten können - und konstatiert, dass das Leben als Aktivität die entscheidende Variable ist, weil es die Substanzströme der Erde neu organisiert und insbesondere die Konzentration von Gasen der Atmosphäre reguliert um sich selbst hervorzubringen und die Bedingungen seiner Genese aufrechtzuerhalten. Leben passt sich demnach nicht vorgängigen Umweltnischen an, wie es die neodarwinistische Sichtweise der natürlichen Selektion vorstellt, sondern bringt Umwelten aktiv, in Ko-Evolution mit hervor. Vgl. James Lovelock, Das Gaia-Prinzip: die Biographie unseres Planeten (Zürich: Artemis \& Winkler, 1991).

39 Greg Hinkle, zit. nach Margulis, Die andere Evolution, S. 8. 
schen Begrifflichkeit von autopoetischer Emergenz. Während Lovelock dem Begriffsschema der Kybernetik erster Ordnung folgt und Gaia damit auf einen Gleichgewichtsdiskurs gründet, ${ }^{40}$ begreift Margulis Gaia im Sinne der Kybernetik zweiter Ordnung als metastabiles System, das ereignishafte Transformationen durchläuft und gerade nicht über ausschließende Grenzziehung zu denken ist, sondern über eine fraktale Topologie der Faltung. ${ }^{41}$ Mit Margulis gedacht, handelt es sich bei Gaia derart nicht um eine Einheit, einen Superorganismus, sondern um eine Mannigfaltigkeit von Ökologien, um eine Ebene der Relationalität aller irdischer Ökosysteme. ${ }^{42}$ Der entscheidende Punkt ist hierbei, dass Gaia hiernach gerade keine Entität darstellt, sondern eine offene und grundlose Aktivität der Relationierung. Wenn Margulis Leben nicht als Seinszustand, sondern als ein »Verb « begreift, ${ }^{43}$ gilt dies auch für Gaia:

40 So stellt Lovelock Gaia in CoEvolution Quarterly (Ableger vom Whole Earth Catalog, einer Plattform in der kybernetische Theorien diskutiert wurden) als ein homöostatisches System vor - »ein kybernetisches System mit einer Neigung zur Homöostase « - und vergleicht derart die lebendige Erde mit einem Thermostat, der Gleichgewicht anstrebt, um eine konstante Temperatur aufrecht zu erhalten. Vgl. Bruce Clarke, »Neocybernetics of Gaia: The Emergence of Second-Order Gaia Theory ", in Gaia in Turmoil: Climate Change, Biodepletion, and Earth Ethics in an Age of Crisis, hg. v. Eileen Crist u. H. Bruce Rinker (Cambridge: MIT Press, 2009), S. 293-314.

41 Gaia ist in Margulis Lesart also metastabil, d.h. "stabil in ihrer reaktionsfreudigen Instabilität «. »Die ganze Erdoberfläche - nicht nur die Lebewesen, sondern auch die Atmosphäre, die wir uns meist als passiven Hintergrund vorstellen - ist so weit vom Gleichgewicht entfernt, dass man am besten das gesamte Äußere unseres Planeten als lebendig betrachtet." (Margulis, Die andere Evolution, S. 153).

42 »Gaia, die lebende Erde, geht weit über jeden einzelnen Organismus [...] hinaus. [...] Das System Gaia erwächst aus mindestens zehn, vielleicht auch mehr als 30 Millionen untereinander verbundener, lebender Arten, die seinen unaufhörlich aktiven Körper bilden [...]. Gaia selbst ist kein Lebewesen, das durch unmittelbare Selektion gegenüber vielen anderen bevorzugt wurde, sondern eine sich entwickelnde Eigenschaft, die aus den Wechselbeziehungen zwischen den Lebewesen, dem kugelförmigen Planeten, auf dem sie zu Hause sind und der Sonne als Energiequelle erwächst. (Ebd., S. 148-49).

Vgl. Lynn Margulis u. Dorion Sagan, Leben. Vom Ursprung zur Vielfalt (Heidelberg: Spektrum Akademischer Verlag, 1997), S. 22: »Die Frage >Was ist Leben?< ist also eine sprachliche Falle. Um sie nach den Regeln der Grammatik zu beantworten, müßte man ein Substantiv nennen, eine Sache. Aber das Leben auf der Erde gleicht eher einem Verb.« 
Gaia ist nicht und sie ist auch nicht eins, sie knüpft nur unaufhörlich ihre Fäden; Gaia nicht als Ganzes, sondern Gaia als immer mitten-drinSein. So lässt sich Margulis Konzeption als fraktale Topologie denken, in dem Sinne, dass sich in jedem Gefüge die multidimensionale Rekursion wiederholt, sodass es kein Innen und kein Außen mehr gibt. Diese Interpretation von Gaia als eine nicht-vereinheitlichende Relationierungsaktivität mobilisiert Bruno Latour als Figur der Medialität der Übersetzung im Rahmen seiner Akteur-Netzwerk-Theorie. Nach Latour würde Gaia demnach gerade nicht »The Whole Earth « bedeuten, die Erde als Ganzes vom Weltraum aus gesehen im Sinne einer Totalität, die dann von außen repräsentierbar wäre, wobei die Perspektivität und Situiertheit dieser Sicht unkenntlich gemacht würde im Sinne des »Göttlichen Tricks «. ${ }^{44}$ Stattdessen impliziere Gaia, dass es keinen neutralen äußeren Beobachter geben kann, bzw. in den Begriffen Haraways, dass der Blick auf die "ganze « Erde durch einen semiotisch-materiellen Apparat hervorgebracht wird, der den Satelliten, die Computer, eine Ökologie von Schaltkreisen, Delegierungen und Übersetzungen einschließt. ${ }^{45} \mathrm{Mit}$ Latour wäre Gaia als Figur von Medialität, Netzwerken und Relationalität zu denken, die mit dem Objektivitätsfantasma und der Unkenntlichmachung von Medialität bricht. ${ }^{46}$ Im Zeitalter des Anthropozäns trete Gaia demnach als Gegenfigur zur modernistischen Naturkonzeption an die Stelle der Natur als Kategorie des ontologisch Gegebenen, Ursprünglichen. Während im modernistischen Schema die Natur als Ort der Nicht-Kultur konstruiert wird, im Sinne einer vorgesellschaftlichen Kategorie als Matrix und Ressource, gebe sich Gaia als durch und durch artifizielles Vernetzen preis, das gerade keine Rückkehr zum Ursprung anzeige, sondern Techniken, Praktiken und Prozesse der Verknüpfung, die keinem (Natur-)Gesetz folgen, deren Regularitäten im Prozess der Verstrickung emergieren ohne auf einen ontologischen Grund oder ein Fundament rückführbar zu sein. Latours Lesart von Gaia als spielerische, weltschöpferische Vernetzung greift damit zurück auf komplexitäts- und chaostheoretische Konzeptionen von Nichtlinearität und

\footnotetext{
44 Vgl. Haraway, Die Neuerfindung der Natur, S. 82.

45 Bruno Latour, »Waiting for Gaia. Composing the common world through art and politics ", a lecture at the French Institute for the launching of SPEAP (London, November 2011), S. 5-7 <http://www.bruno-latour.fr/sites/default/files/124GAIA-LONDON-SPEAP_0.pdf> [Zugriff: 15.03.2015]. 
Emergenz, wonach die Ereignisse der Welt nicht bereits in den Ursachen enthalten sind. Dieser neomaterialistische Einbezug von Nichtlinearität zielt natürlich auf eine Erzählung von Welt als Offenheit und Potentialität, was hierbei mit dem Spielbegriff konvergiert: Welt als spielerische Prozessualität wird gegen die Vorstellung des linear-deterministischen Uhrwerk-Universums gesetzt, dem in der Moderne nur die binär konstruierte Freiheit des Menschen entgegen trat. Die tricksende Gaia nimmt nach Latour hingegen ihren Ausgang in Zonen der Unbestimmtheit und Unvorhersagbarkeit, welche kein intentionales Subjekt mehr benötigen, sondern auf eine mediale Prozesshaftigkeit als kreativem Prinzip des Welt-Werdens verweisen. In dieser neomaterialistischen Lesart hat das irdische Spielen nichts mit kosmischer Harmonie gemein, sondern als »trickster " und »bitch « gründet Gaia auf Zweideutigkeiten und »Betrug «, entfaltet einen »Kakosmos «. ${ }^{47}$

Während für Latour Gaia als Trickstergestalt im Harawayschen Sinne agiert, ${ }^{48}$ ist Haraways Position hier selbst ambivalenter. Während sie in Monströse Versprechen Gaia noch als »Zeichen einer unaufhebbar sozialen Natur « affirmiert, ${ }^{49}$ als jene irdisch-spielerisch-inventive Aktivität, die nicht existiert »vor ihrer Artikulation in heterogenen sozialen Begegnungen, in denen die Akteure keine Menschen und die Menschen nicht 'wir sind ", ${ }^{50}$ distanziert sie sich in When Species Meet von Margulis Gaia-Konzeption. Insofern Margulis Konzeption von Gaia, im Kontrast zur ihrem Konzept von Symbiogenese, auf einer autopoetischen Interpretation von Prozessualität basiert, vermag diese nach Haraway gerade nicht als Beugungsmuster zu agieren, das im »Bauch des Ungeheuers, das manche die Neue Weltordnung AG nennen, geschmiedet " wird, ${ }^{51}$ um eben diese immanent zu unterwandern. »In my view, Margulis and Sagan's symbiogenesis is not really compatible with their theory of autopoesis, and the alternative is not an additive mechanistic theory but a going even more deeply into differentiation. « ${ }^{52}$ Demnach verlangt Haraway eine radikalere Auffassung der fraktalen Topologie des

\footnotetext{
47 Bruno Latour, "Agency at the Time of the Anthropocene ", New Literary History 45 (2014), S. 4.

48 "Yes, She is the perfect trickster. "(Latour, »Waiting for Gaia «, S. 10).

49 Haraway, Monströse Versprechen, S. 58.

50 Ebd., S. 83.

51 Ebd., S. 9.

52 Haraway, When Species Meet, S. 33.
} 
Möbius-Bandes (»a fractal turtling all the way down «) ( $^{53}$ als es die second order-kybernetische Figur von autopoetischer Selbsterzeugung zu leisten vermag. Denn Autopoiesis bleibt letztlich trotz ihrer schwindelerregenden Grundlosigkeit im »Phantasma eines Selben [...], dem es immer gelingt, das Andere zu integrieren ${ }^{54}$ und damit im kybernetischen Dispositiv der Selbstkonstitution ohne Außen. Mit Bezug auf den Biologen Scott Gilbert insistiert Haraway auf einer Verschiebung der Prozesslogik von dem kybernetisch-autopoetischen Modell hin zu einer intra-aktiven, bzw. einer differenzlogischen Differentialität - »figuring relentless otherness «: $:{ }^{55}$ »As I read him, Gilbert's approach is not a holistic systems theory in the sense that Margulis and Sagan lean toward, and his fractal sturtles all the way down arguments do not posit a self-referential unit of differentiation. " ${ }^{56}$ Entgegen der autopoetischen Auslegung von Prozessualität, bejaht Symbiogenese (Selbst-)Alterität, und fungiert damit als Medium für die Harawayschen Figurationen von companion species/ contact zones/jenseitigen Konversationen/Intra-Aktionen, bzw. als kritische Strategie, sich in die Gewebe und Netze des kybernetischen Dispositivs einzuschreiben und diese immanent zu unterwandern. Hierdurch wird auch die Unterscheidung zwischen Symbiose und Symbiogenese hinfällig, da jede symbiotische Beziehung, jede jenseitige Konversation ein Werden-Mit - Symbiogenese - impliziert.

The creative force of symbiosis produced eukaryotic cells from bacteria. Hence all larger organisms - protests, fungi, animals, and plants - originated symbiogenetically. But creation of novelty by symbiosis did not end with the evolution of the earliest nuclear cells. Symbiosis is still everywhere. ${ }^{57}$

Trotz Latours Bemühen Gaia, entgegen des Begehrens nach Ursprünglichkeit, Ganzheit und Unversehrtheit, an die Realität partialer Perspektiven anzubinden, an ein irdisches Spiel, das nichts Unschuldiges oder Konsequenzloses an sich hat, beugt Gaia, in ihrer kybernetischen Logik der Emergenz, mit Haraway gedacht, »die Netze « letztlich vielleicht nicht radikal genug auf die Frage der Verletzbarkeit, Affizierbarkeit und

\footnotetext{
53 Ebd., S. 33.

54 Tiqqun, Kybernetik und Revolte (Zürich: Diaphanes, 2007), S. 19.

55 Haraway, When Species Meet, S. 32.

56 Ebd., S. 33.

57 Margulis, zit. nach Haraway, When Species Meet, S. 31. 
Ausgesetztheit alles Irdischen hin, während dementgegen Symbiogenese gerade nicht »Ordnung aus dem Chaos « impliziert, sondern ein "Werden-Mit « als Affirmation des Nicht-Identischen, der Realität des un/an/ geeigneten Zwischen.

\section{NETZ-SPIELE ZWISCHEN "KRIEG DER STERNE" ${ }^{58}$ UND "RISKY WORLDINGS " 59}

Symbiogenese erscheint bei Haraway als ein Netz- bzw. Fadenspiel des Hervorgehens von Welten, wie das "cat's cradle games in which those who are to be in the world are constituted in intra- and interaction «. ${ }^{60}$ Derart versteht Haraway das »becoming with « symbiogenetischer companion species als irdisches Spiel - und Spiel damit als eine Praxis der Invention aus dem Zwischen, d.h. als jene ereignishafte Relationalität und Medialität, die ihren Termen immer vorausgehen muss und dabei jenseits der Zweck-Mittel-Relation Welten hervorbringt. Entscheidend ist damit die Beziehung zwischen Spiel und Invention, bzw. jener assoziierten Reihe von Begrifflichkeiten der Emergenz, der Öffnung und des Auftauchens, wie Erfindung, Kreativität, Fantasie, Ereignishaftigkeit und schöpferische Potentialität.

Play is the practice that makes us new, that makes us into something that is neither one nor two, that brings us into the open where purposes and functions are given a rest. Strangers in mindful hominid and canid flesh, we play with each other and become significant others to each other. ${ }^{61}$

Dabei unterscheidet Haraway zwischen den Begriffen "game « und "play«: Während game auf vorgängigen Regeln basiert, spielt play mit Regularitäten, indem es sich auf diese bezieht, um sie gleichzeitig aus-zusetzen, und ein Ereignis frei-zu-setzen. Spielen zielt auf ein Aus-setzen von Regeln. Als Beispiel führt Haraway ein transspecies play zwischen einem Hund und einem Esel an: Beide Akteure müssen sich dabei gegen die »Regeln ihrer Art « und das Raubtier-Beute-Verhältnis verhalten um eine noch unbekannte Praxis zu erfinden. ${ }^{62}$ Ihre jenseitige Konversation,

Haraway, Die Neuerfindung der Natur, S. 49.

Haraway, When Species Meet, S. 27.

Ebd., S. 4.

Ebd., S. 237.

62 Vgl. Ebd., S. 233-34.
} 
ihre Kontaktzone, ihr Werden-Mit, das Auftauchen neuer geteilter Welten, folgt gerade nicht dem (Natur)Gesetz, sondern emergiert als ein Muster ohne Grund. Diese schöpferische Dimension des Spiels denkt Haraway über Whiteheads Begriff der Proposition als ein Zusammenbringen des Mannigfaltigen und Heterogenen. ${ }^{63}$ Proposition bedeutet im Sinne Whiteheads keine sprachliche Aussage, sondern verweist auf einen inventiven Modus der Kohärenz- und Konsistenzbildung einer Heterogenität ohne Widerspruch, auf eine maschinische Verbindung im Sinne Deleuzes und Guattaris, derart, dass zum Beispiel im transspecies-Spiel die Geste des Beißens mit Freude statt mit Aggression verknüpft wird, ohne dass es hier einen Widerspruch gäbe.

Inwiefern ereignet sich in dieser Affirmation von Kreativität in den neomaterialistischen Konzeptionen eines irdischen Spiels (earthly play) nun eine Subversion des kybernetischen Machtregimes? Insofern das kybernetische Kontrolldispositiv in den 1980er Jahren von der Systemmetapher zum Paradigma des (dezentralisierten) Netzes wechselte, sich derart auf der universellen Vernetzung gründend, der ständigen Bewegung als (Waren- und Informations-)Zirkulation als ein wucherndes Dispositiv ohne Außen ausbreitet, hat es die Hervorbringung des Neuen seinen Machtoperationen immanent gemacht. Wie lässt sich vor dem Hintergrund dieses kybernetischen Kapitalismus und seiner Kontrolldispositive der Vernetzung, welche der Logik der unendlichen Invention und Kreativität, des Fließens und der Ströme, der unendlichen Bewegung folgen, überhaupt weiterhin auf einem differentiellen Einsatz eines neomaterialistischen Spielbegriffs insistieren? ${ }^{64}$ Entscheidend ist hier meiner These zufolge die Konzeptualisierung des Verhältnisses zwischen Emergenz, Kontingenz und Prekärsein. So muss die Kybernetik selbst vor dem Hintergrund jener "probabilistischen Revolution « gedacht werden, die es nicht mehr zuließ am Grund der Natur ein starres Regelwerk ewiger Gesetze anzunehmen, das positivistisch durch die Wissenschaften aufgedeckt werden würde, sondern die dementgegen den Zufall als Verhalten-

63 Vgl. Ebd., S. 243.

64 Vgl. Tiqqun, Kybernetik und Revolte, S. 40: "Die Kybernetik ist folglich darauf ausgerichtet, zu beunruhigen und im gleichen Zuge zu kontrollieren. [...] Der Notstand, der das wesentliche Merkmal der Krisen ist, ermöglicht es, die Selbstregulierung anzukurbeln, sich selbst als permanente Bewegung in Gang zu halten. Anders als bei der klassischen Ökonomie [...], ist es umgekehrt nun das >Wachstum`, das ein grenzenloser Weg zum Gleichgewicht ist. « 
sprinzip der Natur zu denken gezwungen war. ${ }^{65}$ Aus dieser Situation ergibt sich das (in sich gespaltene) kybernetische Begehren nach Steuerung ausgehend vom ungesteuerten Zufall, d.h. das "praktische Problem der Beherrschung von Unsicherheitsfaktoren « und das » metaphysische Problem der Begründung der Ordnung ausgehend von der Unordnung ", um sowohl theoretisch als auch technologisch »das Gesetz ausgehend vom Chaos und das Gewisse ausgehend vom Wahrscheinlichen neu zu definieren «. ${ }^{66}$ In dieser Umwandlung und Bändigung von Kontingenz in Wahrscheinlichkeit gründet auch die von Neumann und Morgenstern entwickelte "Spieltheorie « der Kybernetik, die davon ausgeht, dass das wahre Gesetz der politischen Ökonomie der Zufall ist. ${ }^{67}$

Entgegen einer einfachen Kurzschaltung von Vernetzung, Spiel und Kreativität besteht Haraways diffraktioneller Einsatz darin, Spielen an eine Figur des Hervorgehens von Welten, bzw. der Emergenz zu binden, die mit Kontingenz zusammenfällt, aber gerade nicht als deren Überwindung, d.h. nicht im kybernetischen Sinne einer »Ordnung aus dem Chaos ", sondern als Affirmation der Kontingenz, des Nicht-Identischen, der Realität des unangeeigneten Zwischen. Haraway spielt mit der kybernetischen Eingemeindung von Potentialität und Kreativität, indem sie einer Strategie der Affirmation der Endlichkeit und des Prekärseins der verstrickten Welten folgt. So entkoppelt sie den Spielbegriff als schöpferische Aktivität des Zwischen von der Transzendenz der kybernetischen Schöpfungslogik, welche letztlich innerhalb des Dualismus von Form und Materie, Denken und Körper darauf zielt, ganz im Sinne von Hans Moravecs Mind Children, den entkörperten »Geist « im Cyberspace zu verewigen und $\mathrm{zu}$ transzendieren. ${ }^{68}$ "Besides, it is not potentially infinite expressiveness that is interesting for play partners but, rather, unexpected and nonteleological inventions that can take mortal shape only within the finite and dissimilar naturalcultural repertoires of companion species. " ${ }^{69}$ Nach Haraway geht es beim earthly play gerade nicht um unendliche Schöpfung, sondern um »inventions that

\footnotetext{
65 Vgl. Astrid Deuber-Mankowsky, Praktiken der Illusion. Kant, Nietzsche, Cohen, Benjamin bis Donna J. Haraway (Berlin: Verlag Vorwerk 8, 2007), S. 311-27.

66 Tiqqun, Kybernetik und Revolte, S. 21.

67 Vgl. ebd., S. 35.

68 Vgl. Hans Moravec, Mind Children (Hamburg: Hoffmann und Campe, 1990).

69 Haraway, When Species Meet, S. 237, m.H.
} 
can take mortal shape " ${ }^{70}$ d.h. um Praktiken, die zugleich öffnen, als auch die Sterblichkeit und Ausgesetztheit ihrer Existenz bejahen. Earthly play wäre damit als jene Bewegung des sich-Ereignens zu denken, die eben keine Verewigung im technohumanistischen Himmel des Immateriellen-Göttlichen-Reinen darstellt, sondern die ihre eigene Endlichkeit, Verletzbarkeit, Sterblichkeit, Ausgesetztheit unbedingt bejaht. Damit unterwandert Haraway die kybernetische Logik der Verleugnung von Materialität und Prekärsein, insofern ihr Werden-Mit keinerlei Transzendenzbestreben folgt, sondern eine Prozesshaftigkeit von Materialität zu denken gibt, die einerseits der kybernetischen De-Essentialisierung und De-Substanzialisierung folgt, diese andererseits »beugt ", indem die Prozessmaterien an ihre Sterblichkeit gebunden werden. »How is >becoming with a practice of becoming worldly? [...] I am a creature of the mud, not the sky. «71 Entgegen der erhabenen Auflösung ins Virtuelle setzt Haraway hier die (technologisch-organisch-hybriden) prekären Kontakt-Zonen, "mortal world-making entanglements that I call contact zones «. ${ }^{72}$ Ihr geht es um die Verantwortung, aus der diese Begegnungen gestrickt sind. Die Partner (»ordinary beings-in-encounter « $)^{73}$ gehen hier ihrer Begegnung nicht voraus, die pluralen, anderen, wirklichen Welten gehen in Intra-Aktionen hervor, sind aber hierin zugleich ausgesetzt, das heißt sie stehen ernstlich auf dem Spiel. Indem Haraway die kybernetisierten Netz-Welten durch ein im-Dreck-Spielen (»playing in the mud « $)^{74}$ beugt, gibt sie quer zur technohumanistisch-kybernetischen Vision einer "autre-mondalisation ${ }^{75}$ und "alter-globalization ${ }^{76}$ statt. Relationalität fällt derart nicht in eins mit Komplexität(ssteigerung), sondern bedeutet "Sorge um « die prekären Begegnungen anderer Welten. » The point is not to celebrate complexity, but to become wordly and to respond. $" 77$

In der finalen Szene von Zoo Citys Plot inszeniert Huron, ein einflussreicher Musikproduzent Johannesburgs, der sein Getiert-Sein bis

\footnotetext{
Ebd.

Ebd., S. 3.

Ebd., S. 4.

Ebd., S. 5.

Ebd., S. 30.

Ebd., S. 3.

Ebd.

77 Ebd., S. 41.
} 
dato geheim halten konnte, ein blutiges Spiel, das darauf zielt, aus der symbiotischen Beziehung zu seinem Tier heraus zu kommen, das prekäre Spiel selbst zu beenden, seine existenzielle Ausgesetztheit auf eine Nichtverletzbarkeit hin zu transzendieren. So versucht Huron die magische Verbindung, die zwischen ihm und einem Albino-Krokodil besteht, auf einen Jungen ( $\mathrm{S}$ 'bu) zu übertragen, der dazu seine Schwester (Songweza) töten muss, um im Anschluss befreit, gereinigt, ungetiert, das Krokodil letztlich selbst ermorden - und in Stücke zerhackt für viel Geld als Muti verkaufen - zu können.

Hurons Stimme liegt über dem Wasser. 'Kinder, das hier ist mein Freund, Herr Krokodil. Sagt guten Tag, Herr Krokodil. Er möchte auch gern euer Freund sein. Ein ganz besonderer Freund. Weil, ganz ehrlich, ich hab das Ding satt bis auf den Tod. ‘ [...] >Ich will nicht<, sagt Songweza in einer Kleinmädchenstimme am anderen Ende der Höhle. [...]

,Wir tun alle manchmal Dinge, die wir nicht tun wollen`, sagt Huron. `Es ist wie ein Spiel.

'Wie >Blood Skies«, fragt S'bu. Seine Stimme klingt fern und vage, wie das Echo eines menschlichen Wesens.

'Ich weiß nicht, was das ist $`$ blafft Huron.

‘Ein Videospiel.

'Ja, es ist genau wie ein Videospiel.< Jetzt hat Huron den Tonfall eines Verkäufers.

'Kooperativ oder non-kooperativ?

>Definitiv nicht kooperativ. ${ }^{78}$

Hurons Spiel ist ein männliches »Krieg der Sterne «-Spiel der »göttlichtranszendenten « Überwindung allen irdischen Spielens: Er spielt, um aus dem Spielen-Mit, aus der Verstrickung, aus dem Prekärsein des Spiels selbst heraus zu kommen. Zinzi und Faultier, die ihren Geliebten Benoit und seinen Mungo in ihr gefährdetes Spiel verstrickt haben, kommen aus dem Spiel, aus den niemals unschuldigen Verstrickungen, nicht raus. Benoit liegt am Ende des Buches im ungewissen Zustand des Komas, während der Mungo abgemagert und elend die Krankenhausflure aufund abrast. Zinzi und Faultier ziehen mit "einer rot-blau karierten Flüchtlingstasche voller Falschgeld « ${ }^{79}$ gen Kongo um Benoits Ehefrau und Kinder Asylantragsformulare für Südafrika zu besorgen. »Die

78 Beukes, Zoo City, S. 341.

79 Ebd., S. 358. 
Begegnung wird beklemmend sein. [...] Und danach? ${ }^{80}$ Nichts löst sich auf in Zoo City. Es gibt kein Jenseits, keine Rettung, keine Verdammnis, nur ein symbiogenetisches, organisch-technologisches Geflecht, das von den verbindlichen Verpflichtungen von Verschränkungen handelt $»$ figures of mortal relatedness ${ }^{81}$.

Das earthly play, das irdische Spiel, ist in den »intensivierten, gefährlichen und fruchtbaren Fusionen « dieser hybriden Netze zu denken, ${ }^{82}$ hier und jetzt, dieses "Anderswo $",{ }^{83}$ in den un/an/geeigneten Zwischenzonen der Unbestimmtheit der verstrickten Welten. Derart fällt das irdische Spiel nicht in eins mit dem kybernetischen Dogma des Neuen und der Kreativität als Gesetz des Universums, sondern situiert sich als ein »concern for « im Sinne Whiteheads, das heißt »es geht hier ernstlich um « uns irdische »critter ", ${ }^{84}$ um »eine Gemeinschaft derer, die nichts gemein haben «, ${ }^{85}$ um jene Unbestimmtheitszone von Prekärsein und Ereignis, um eine Affirmation dieses endlich-irdisch-sterblichen Spielens selbst. »Queer messmates in mortal play, indeed. $\ll^{86}$

Ebd., S. 359.

Haraway, When Species Meet, S. 12.

Haraway, Monströse Versprechen, S. 10.

Ebd., S. 11.

Ebd., S. 5.

85 Im Sinne von Alphonso Lingis, Community of Those Who Have Nothing in Common (Bloomington: Indiana University Press, 1994).

86 Haraway, When Species Meet, S. 19. 


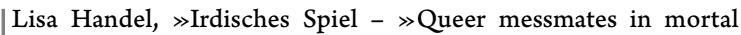
play ««, in Denkweisen des Spiels: Medienphilosophische Annäherungen, hg. v. Astrid Deuber-Mankowsky und Reinhold Görling, Cultural Inquiry, 10 (Wien: Turia + Kant, 2017), S. 191-212 <https://doi.org/10.37050/ci-10_11>

\section{QUELLENANGABEN}

Beukes, Lauren, Zoo City (Reinbek bei Hamburg: Rowohlt Taschenbuch Verlag, 2015)

Clarke, Bruce, »Neocybernetics of Gaia: The Emergence of Second-Order Gaia Theor $y \ll$, in Gaia in Turmoil: Climate Change, Biodepletion, and Earth Ethics in an Age of Crisis, hg. v. Eileen Crist u. H. Bruce Rinker (Cambridge: MIT Press, 2009), S. 293-314

Deleuze, Gilles und Félix Guattari, Tausend Plateaus. Kapitalismus und Schizophrenie (Berlin: Merve Verlag, 1997)

Deuber-Mankowsky, Astrid, Praktiken der Illusion. Kant, Nietzsche, Cohen, Benjamin bis Donna J. Haraway (Berlin: Verlag Vorwerk 8, 2007)

Haraway, Donna, Monströse Versprechen. Die Gender- und Technologie-Essays (Hamburg: Augment Verlag, 2006)

—Die Neuerfindung der Natur. Primaten, Cyborgs und Frauen (Frankfurt a.M.: Campus Verlag, 1995)

When Species Meet (Minneapolis: University of Minnesota Press, 2008)

Jones, Gwyneth, »Zoo City by Lauren Beukes - review « (2011) <http://www.theguardian.com/ books/2011/may/14/zoo-city-lauren-beukes-review> [Zugriff: 10.08.2015]

Latour, Bruno, »Agency at the Time of the Anthropocene «, New Literary History 45 (2014), S. 4 $<$ https://doi.org/10.1353/nlh.2014.0003>

— $\gg$ Waiting for Gaia. Composing the common world through art and politics $\ll$, a lecture at the French Institute for the launching of SPEAP (London, November 2011), S. 5-7 <http://www.bruno-latour.fr/sites/default/files/124-GAIA-LONDON-SPEAP_ 0.pdf> [Zugriff: 15.03.2015]

Lingis, Alphonso, Community of Those Who Have Nothing in Common (Bloomington: Indiana University Press, 1994)

Margulis, Lynn, Die andere Evolution (Heidelberg: Spektrum Akademischer Verlag, 1999)

Moravec, Hans, Mind Children (Hamburg: Hoffmann und Campe, 1990)

Tiqqun, Kybernetik und Revolte (Zürich: Diaphanes, 2007) 